\title{
LA CONTRIBUTION DES JEUX AU DÉVELOPPEMENT DES LETTRES ET DES ARTS EN GRÈCE ANCIENNE*
}

\author{
Nikolaos Yalouns \\ Ancien Directeur du Service des Antiquités et du \\ Musée National d'Athènes
}

Résumé:

L'importance de l'athlétisme dans la vie courante n'a jamais été aussi forte qu'en Grece ancienne, à cause de son rapport avec la religion et du poids de. l'idéologie de la compétition, de l'agonistique: Les concours sportifs non seulement prenaient place lors des fetes religieuses, mais leur siege se trouvait auprès des temples et des grands sanctuarires. Les diverses modalités de compétition étalent censées avoir été instituées par les dleux eux-mémes. D'autre part, les rapports avec les jeux funéraires sont évidents, ainsi qu'avec d'autres ittuels, tels le choix de l'époux ou la sucession au thróne. L'émula tion, par ailleurs, était a la racine du concept d'excellence. Pidéal de plénitude de l'homme grec l'exploit (physique et intellectuel) était capable de distinguer l'homme rationnel de la nature sauvage et illogique Agon (combat) et Nike (victoire) ont été personnifiés de bonne heure. I agon étalt la sève de l'activité des athlètes mais également des poètes. orateurs. musiciens, sculpteurs, peintres, córamistes etc. ies. lieux d'entrainement (palestres et gymnases) sont vite devenus de véritables centres d'education géne. rale. Enfin, le milieu sportif a fourni à la littérature et a l'art grec l'ispiration, les sujets et les modèles dont ils se sont nourris pendant toute leur histoire.

(')Conférence fait à Săo Paulo, au Museu de Arqueologia e Etnologia USP, en septembre 1984. 
L'athlétisme n'a jamais été plus étroitement lié à l'art du monde ancien ou moderne qu'il ne l'a été à l'art de la Grèce ancienne. Notons même que dans l'art moderne, l'exercice sportif a particulièrement peu inspiré les artistes contemporains.

Nombreuses sont les raisons auxquelles est attribuable cette position particulière occupée par l'art grec ancien. Deux en sont cependant les principales: son lien étroit avec la religion, source principale d'inspiration des artistes grecs, et la place prédominante et centrale occupée par lidéal de lexercice athlétique dans la vie des Grecs.

La relation entre l'exercice athlétique et la religion fut três étroite. Aucune fête religieuse et aucun sanctuaire important n'existaient en Grèce, sans associer Padoration avec l'organisation des Jeux.

Ce n'est pas un hasard si les premiers endroits destinés aux compétitions étaient situés dans les cours et aux alentours des temples, avec les dieux comme spectateurs principaux. C'est d'ailleurs aux dieux et aux héros qu'était atribuée la fondation des Jeux. Ces Jeux étaitent organisés en leur honneur dans touts les grands sanctuaires panhelléniques, ainsi que dans une multitude d'autres moins importants, disséminés sur tout le territoire hellénique. Les Grecs attribuaient égalemente aux dieux et aux héros mythiques la conception même des divers jeux athlétiques et ils considéraient plusieurs parmi eux comme les premiers vainqueurs.

Ainsi, selon eux, le pugilat a été inventé par Apollon qui a vaincu Arès dan cet exercice, tandis qu'à l'épreuve de la course, Apollon a vaincu Hermès. Selon une autre tradition, Zeus a de noveau gagné Cronos à la lutte. Héraclès a amené ses cinq frères à Olympie, les Dactyles-Courètes, pour y courir et il a couronné pour la première fois le vainqueur avec une branche d'olivier sauvage, le "cotinos". Enfin, Jason a inventé le pentathlon au cours de Pexpédition des Argonautes.

Selon un autre récit mythique, c'est le sage Centaure de Pélion, Chiron, pédagogue d'Achille, qui a institué la culture physique. Chiron combinait la médecine avec la gymnastique, par laquelle il accordait aux jeunes héros la beauté et la vaillance.

C'est de la religion grecque $\in t$ de la mythologie qu'est donc issu Pesprit competitif, qui avait fasciné la pensée et le coeur des Grecs. Leur soif du concours se reflète non seulement dans les jeux athlétiques pacifiques des dieux et héros, mais également par leurs combats contre la nature sauvage et illogique, symbolisée par les Géants, les Titans, les Centaures et les Amazonas. La Gigantomachie, le combat inexorable entre les dieux olympiens et les Géants ou les Titans, d'une part, et la lutte des héros grecs contre les Centaures et les Amazones, d'autre part, (la Centauromachie et l'Amazonomachie), sont devenues des symboles et des modeles pour chaque compétition des Grecs, notamment lors de leurs heurts avec les barbares. L'assimilation de Phomme avec les héros et les dieux, fondateurs des jeux, était la mission principale de chaque sanctuaire. Leur misson spirituelle était d'enseigner que seul l'exploit permet à Phomme de s'affranchir de la "vie sauvage", "theriandes bios", d'éveiller et développer ses capacités psychiquesi et physiques inépuisables ainsi que les vertus, que lui a données la nature, a fin de devenir libre. Le contraire, la paresse et pinaction conduisent à lesclavage et à la servitude "doulikótata tón estin tô tryphån" enseignaient les anciens. 
Par conséquent, les Jeux se déroulant dans les I sanctuaires n'étalent pas un simple spectacle, mais une cérémonie sacrée, d'autant plus d’ailleurs que la gymnastique et la musique étaient, d'aprés Platon, des dons des dieux.

Les vainqueurs dans les sanctuaires Panhelléniques devenaient, d'une certaine manière, les associess de Péclat divin et de la vie intemporelle des premiers vainqueurs mythiques; c'est la raison pour laquelle plusieurs parml eux ont été adorés après leur mort comme des heros dans leurs villes natales. La victolre aux Jeux constitualt le plus grand bien et le plus grand honneur, auquel pouvalt aspirer le commun des mortels.

Les Jeux amènent la consécration de Pidéal de la noble émulation, qui a constitué le fondement de l'éducation des jeunes dans toutes les villes helléniques et le stimulant principal pour la culture et le dévebppement maxtmum ldes aptitudes physiques, psychiques et intellectuelles de lhomme. Selon la conception hellénique toute grande oeuvre est le résultat d'une émulation.

"Aién aristéuein kal ypelrochon émmenal állon" ("la quête constante de la perfection et du premier rang") est la règle proposée par Hippolochos à son fils Glaucos, lorsque celui-cl part pour Trole. C'est sur ce même principe que se fondainent les paroles de Pélée envers son fils, Achille, également au départ de ce demier pour Troie.

Cette très célèbre incitation, formulée d'une manière si dense et comme une sentence dans le plus ancien texte du monde hellénique (et européen) - l'épopée homérique, éclaire la conduite et les idéaux des héros homériques mais également de tous les Grecs des temps historiques.

Tout comme les héros homériques "exceilent" tous les jours ao combat mais également aux épreuves athiétiques, étant organisés pendant les heures libres, de la même manière les Grecs des temps historiques ont comme idéal "l'excellence" dans la competition, dans le cadre des Jeux et aussi dans toute mánifestation de läctivilté humaine.

C'est la ralson pour laquelie, brsqu'après la grandiose victolre des Grecs contre les Perses, les Grecs se sont à nouveau rassemblés en 476 à Olymple, avec leur fol accroe en lídéal de l'homme lbre, les spectateur's ont oublié ceux qul concouralent sur la plste, car là se trouvalt Partisan de la victoire contre les Perses, Thémistocle. C'est lul que les spectateurs acclamalent comme vainqueur olymplque, et c'est sur lul que les regards étaient fixés pendant tout la joumée, dit Plutarque.

Nul autre peuple, avant et apres les Grecs, ne s'est proposé un tel but, Pexplott, ot avec une telle exclusivité et une telle intensité; et chez nul autre peuple, la récompense de cet idéal, la simple courome de la victoire, la branche d'ollvier sauvage, n'a constitú́ de manlère aussi constante et soutenue, le bien suprême entre tous les trésors du monde.

Dieux et héros sont présentés dans la mythiologle grecque comme les "premiers blenfalteurs" ou come les "premiers iniciateurs" de diverses inventions, non seulement en matière d" exercice physique mais aussi dans les oeuvres de la civilisation intellectuelle et matérielle. Par exemple, Athéna a enseigné la course des chars, Bellérophon a dompté le premler le cheval, Palamède a enseigné la médecine, Aristé la culture de la terrie. 
L'esprit de Pémulation s'étend également aux occupations rurales quotidiennes: labourage, moisson, vendange. Lityerse, personnage mythique, fils du roi Midas, demandait à quiconque était de passage dans son royaume au temps de la moisson de se mesurer avec lul au moissonage.

Le choix de l'époux est souvent opéré par concours athlétiques. Atalante avait décidé qu'elle épouserait celui qui la vaincrait à la course. Après plusieurs prétendants ayant échoué à la dépasser, elle a enfin épousé Hippomène qui seul avait été plus rapide qu'elle. De même, Ulysse a épousé Pénélope après avoir auparavant gagné les autres prétendants à Pepreuve de la course. Quelque chose d'analogue est advenue dans le cas de Pélops également; il a épousé Hippodamée après avoir auparavant vaincu son père, Oenomaos, à la courses de chars.

Dans d'autres cas, l'épreuve dans un concours détermine la succession au trône. Scythe, fils d'Héraclès et Echidne, dont les Scythes portent le nom, est monté sur le tróne aprés avoir vaincu à Parc ses deux frères conformément aux conditions posées par șon père.

L'exercice athlétique et Pespirit d'émulation prédominaient dans chaque cité grecque ancienne comme son souci principal et son activité primordiale. Dans la période de paix, les hommes jeunes et múrs passaient leur vie dans les Palestres et les Gymnases, lieux d'exercice quotidien du corps, mais égalemente de l'esprit. En même temps que Pexercice physique, on cultivait égalemente dans les Gymnases et les Palestres la modestie, la plus importante vertu du jeune athlète, dont l'opposé était l'arrogance (orgueil, l'hybris). Cette tåche était confiée à des citoyens respectables et sages, les gymnasiarques, ultérieurement appéles "kosmetal".

Très vite ces lieux, Gimnases et Palestres. ont évolué en centres d'éducation générale, où l'exercice du corps avait leu dans le cadre d'un programme plus général de culture de l'esprit et de l'âme de jeunes gens. Il est caractéristique qu'Aristote a enseigné dans un Gimnase, le Lycée d'Athenes. Nous savons en outre que le Gymnase de Ptolémée à Alexandrie possédait une riche bibliothèque et salle de lecture, où des philosophes enseignaient jusqu'au temps de Cicéron. Même aujourd'hui, dans beaucoup des pays, les écoles secondaires s'appelent Gymnases et Lycées.

Pendant des concours athlétiques dans les grands centres religleux (Olympie, Isthme, Némée et Delphes) ou pendant des conflits guerriers, les cités étalent fières de leurs valllants jeunes hommes, qui revenaint couronnés pour leur victoire sur la piste ou le champ du combat. C'est l'époque, où il n'y avait pas d'amis du spot, car tous étaient des athlètes; présent, très peu nombreux sont ceux qui pratiquent un sport et tous les autres sont les admirateurs de ces champions, les amis du sport.

Le séjour permanent dans la Palestre et Pexercice continu amenèrent très vite les athlètes dès vers la fin du Vill ême siècle av. J.C. à s'entraîner entièrement nus (gymnós). Cette nudité-gymnótes-fut d'ailleurs si intimement liée à l'exercice physique que le terme "gymnastique" en est issu ainsi que le lieu où se déroulaient les exercices, $1 \theta$ Gymnase.

Dès lors, un idéal de la forme masculine est crée. Il est repésenté dans une nudité héroïque, au moment de l'épanouissement suprême corporeil et mental de l'homme, 
c'est-à-dire lorsqu'il est adolescent (éphèbe) et homme en même temps, au moment même où il passe d'un âge à l'autre. C'est "phomme-ephèbe" (andrópais). Non seuloment les mortais mais également les héros et les dieux étaient représentés sous cette forme idéale de l'andrópais dès le début de la sculpture hellénique (VII ème siècle av. J.C.) jusqu'aux temps classiques avancés (V ème -IV ème siècles av. J.C.).

Les Gymnases et les Palestres remplis par les corps vigoureux des jeunes qui s'y exerçaient, constituaient une riche source d'inspiration pour les artistes, sculpteurs et peintres. Ces leux leur ont fourni une chance unique pour étudier les corps des athites bien bâtis et musclés grâce à l'exercice; ce sont ces corps d'athlètes qui ont donné des milliers de chefs d'oeurre.

Après les guerres médiques, l'exercice physique prédomine tellement dans les pensées des Grecs, que même les dieux, lorquitis ne sont pas représentés dans des scènes de compétition, (par exemple, la Gigantomachie), sont figurés dans des positions s'inspirant des scènes de la Palestre (Poséidon d'Arteimision du Musée National d'Athènes inspirée par la position d'un athlète qui lance le javelot). Et bien plus, les dieux sont représentés comme des jeunes hommes robustes grâce à l'exercice, comme s'il n'existait pas d’âge mâr. Les dieux figurent avec la fraicheur et la sveltesse de la jeunesse imperissable.

Volla donc pourquol Part grec abonde en sujets innombrables, pris dans la vie de la Palestre, du Gymnase, des concours athlétiques, des combats et, pár extension, des exploits des héros et des dieux. Dieux, héros et mortels s'exercent, et leurs victories figurent sur toute sorte d'oeuvre d'art, en sculpture, travail du métal, peinture de vase et dans la décoration des temples, de sanctuaires et des cités.

Les exemples les plus llustres et plus célèbres dans le monde entier sont bien sâr les deux compositions des deux frontons du temple de Zeus à Olympie (combat des lapthes contre les Centaures et concours des chars entre Pélops et Oinomaos), ainsi que le fronton ouest du Parthenon (concours entre Athéna et Poséidon pour le patronage de la ville d'Athènes). Mais aussi la frise de ce meme temple: cette demière immortalise la procession des Panathénées, organisée tous les quatre ans à Athènes, avec des concours gymniques et musicaux, en Phonneur d'Athéna, patronne de la cité. Sur la fritse du temple, dieux, héros et citoyens athéniens se rencontrent sur la mêrne zone. Les premiers assis suivent avec plaisir la procession, organisee en leur honneur par Pélite de la jeunesse athénienne, à cheval ou sur des chars, des prêtres et prêtresses, des vieillards, tous profondément recueillis.

On pourrait donc affirmer à juste titre que l'athlétisme constitue l'objet et même la source d'inspiration principale de lart grec et que Phistoire de ce dernier s'identifie dans une très grande partie avec l'ancienne agonistique. C'est la raison pour laquelle l'histoire de l'art ancien a essentiellement comme objet d'étude les statues des athlètes. Cela est surtout valable pour Pépoque archaïque et classique (600-323 av. J.C.), lorsque seuls les vainqueurs avaient le droit d'avoir leur statue dans les sanctuaires ou les cités. Très peu nombreuses sont les statues d'autres mortels qui ont été érige日s au cours de cette période, et cela dans des cas absolument exceptionnels. A Olymple, il y avait des mit- 
liers de statues d'athètes vainqueurs, mais les autres ex-votos aussi étaient toujours au molns lés à ragonistique.

Et ce n'est pas uniquement Phistoire de lart grec qui est exprimee par les offrandes innombrables des vainqueurs existant dans les anciens sanctuaires. Toute l'histoire grecque se trouve concentrée dans les grands sanctuaires panhelléniques, où les cltés Immortallsaient les grands évènements de leur histoire, au moyen d'offrandes pleuses de reconnalssance.

Chaque grand sanctuatre étalt des archives en pierre; un livre ouvert où le visiteur ancien lisalt sur les inscriptions des offrandes toute l'histoire ancienne.

La contribution du sanctuaire d'Olymple, du plus ancien sanctuaire de Grèce, est doailleurs particulìrement importante, car c'est de là que provient Pétablissement de lordre chronologlque unlforme des évènements historiques de PAntiquité, tandis qu'auparavant, chaque région possédailt son propre calendrier local.

Par la reconnalssance de la sucession des Olympiades comme un calendrier panhellénique dans tout le monde ancien, la première Olympiade en 776 av. J.C. a consttué le jalon entre l'époque protohistorlque et historique ainsi que le début de l'ordre chronologique des évènements de toute Phistoire ultérieure. Le besoin et le sens de l'exercice athletique et de la compétition, dans la vie quotidienne ainsi que dans les grands moments de l'histoire grecque étaient si profonds que déjầ au début du $V$ ème siècle av. J.C. la compétition $(A g 6 n)$ avalt pris une forme concrète, et sa statue étalt dressée dans le sanctuaire d'Olymple, avec des haltères dans les mains (Paus. 5,26,3, oeuvre perdue de Dionysos d'Argos). Une autre représentation en rellef du même personnage décoralt la table en ivoire et or, sur laquelle étalent déposées des couronnes destinées aux valnqueurs ollmplques (Paus, 5,20,2-3). L'Agón était également représenté comme Pemblème des pléces de monnale de plie de Péparithos (actuellement Skopélos).

C'est ce même esprit d'emulation et de compétition, qui inondait les Grecs qui très vite à donnée à la Victoire (NikE) une forme concrète et tanglble. Les innombrables représentations de la déesse dans des couvres de sculpture et de peinture ainsl que dans los arts mineurs révèlent sa présence incessante dans la pensée et les coeurs des Grecs. L'esprtt compéttif et Pesprit d'émulation se sont également étendus dès les tempos les plus éloignés déjà à toute autre manifestation créatrice de l'homme. Ce fut le factour essentiel de rélaboration de la civilisation grecque dans son ensemble, car c'est a ce factour compéttif qu'est attribuée toute réalisation importante, non seulement en matiere deexercice athlétlque mais egalement dans les lettres, les arts, la politique et toute autre manifestation de la vie.

Dans les Jeux funératres pour lo rol défunt de Amphidamas, Hésiode gagne le prix pour son chant et acquiert alnsi, b premier dans Phistolie de Pesprit et de l'art, la victore et la récompense dans Parène artistique.

Mals, en outre, des concours de danse sont déjà organisés à Pépoque géométrique (750 av. J.C.). Une oenochos attlque, gracleuse et simple de cette époque, porte une inscription sur ses épaules (fig. 1) ${ }^{(+)}$. Outre le fait que cette inscription constitue un

(+) - "(ce vase) dolt être donné à celul parml les danseurs qui avec plus de grace et d'habilite". 
des très anciens spéctmens de Palphabet grec, elle nous informe que ce vase a êté offert pendant un concours de danse à celul qui dansalt avec phus de grâce et plus de légèreté ("os nan orchestón pánton atalótata palzep").

Au début du vème siècle av. J.C., deux grands peintres de vase de l'Attique rivalisent pour mieux dessiner. Euphronios est fier de son dessin sur une coupe, dès qu'il Pa terminé, et il écrit sur un côté du vase: "Euphronios a peint, admirez donc"; et son rival contemporain, s'adonnant au meme art, écrt sur une de ses compositions de peinture sur un autre vase: "Euthymides a peint comme jamais n'a pas pu Euphronios"). Le falt qu'un tel comportement ne consthue pas une particulartie personnelle d'un artiste mais que cette inscription, arrivee jusqu'à nous, exprime la disposition compéttive habituelle entre collegues, est souligné de la manière la plus vive par lancien proverbe: "Kerameus Keramel kotéi" ("Kéramé s'oppose à Kéramée"). Mais la concurrence dans le domaine artistique ne se limitait pas seulement aux peintres de vases. Elle est également consciente et vive au temps archaïque et classique entre les autres créateurs, poètes et artistes. Les annés, nous dira Pindare, n'arriveront pas à effacer son ceuvre poétique, comme ce sera le cas des oeuvres des statuatres.

Cet espitt compétitif profondément ancré chez les Grecs, qul a constitué le stimulant pour les réalisations guerrières comme pour les conquetes paclíques, ne s'est par manifesté seulement dans leur vie privê, mais a souvent pris une forme organisê sur laquelle se fondent des manifestations et creations à buts culturels de blen plus grande portee. On ne salt peut-itre pas assez que très souvent la création d'importantes oeuvres artistiques était adjugée à la suite de concours artistiques, où concurraient des artistes de premier rang venus de diverses régions du monde hellénique. Um témolgnage ancien nous informe que Phidlas a révêlé ses capacités uniques lors d'un tel concours, car il avait tenu compte de la déformation optique, que subirait le visage de la statue d'Athéna, lorsqu'elle serait placée sur un socle très elevé, et il l'avait ainsi fabriqué suivant des proportions modifiés, ce qui lui valut ladmiration du jury et la victoire. En outre, ont été sauvées les quatres coples romaines des Amazones, toutes oeuvres de célebres artistes des temps classiques: Phldias (fig. 2), Kreslas, Phradmon et Polycleto (fig. 3), pour participer au concours lancé à Ephèse.

Et enfin, Pinscription figurant sur la base de la célebre Victoire de Péonios a Olympie (f. 4) ("Paiónios epofese Mendaíos, ós kai taakrotéria poión kal tón naon enika), nous fourntt un témoignage certain d'un tel concours.

Mais, la tendance compéttive a culminé et a incontestablement contribué a la création d'couvres intellectuelles avec l'insttution des représentations dramatiques lors des fêtes de Dionysos a Athènes. Les poètes tragiques présentalent alors leurs tótralogles (trols tragedies ayant un sujet central et un drame satyrlque) devant le public athenien, en attendant la décision du jury pour la réconpense. C'est dans une telle perspective que les poettes tragiques on écrt leurs oeuvres et c'est dans ces conditions dificlies de compétition que se sont révélés les trols prestigleux auteurs tragiques, Eschyle, Sophocle, Euripido.

Plus tard, au IVerme slècle av. J. C., doux autres géants de la parole, partictpent à la compétition devant le dème des Athéniens, Démosthène et Eschine. 
Par la magie et Péloquence de sa parole, Démosthène gagne la voix des Athéniens tandis que son adversaire, Eschine part en exil.

Lorsque plus tard ce dernier est prié et lit son discours devant un autre auditoire à Rhodes, où il vivait en exil, et l'assistance s'emporte contre les Athéniens, qui n'avaient pas été émus par le discours élaboré de l'orateur, Eschine, comme un athlète authentique, répondit. "Oh, et si vous aviez ecouté te discours de ce fameux adversaire, vous ne seriez pas surpris de sa victoire".

L'esprit agonistique et compétitif s'étend sur toute l'étendue de la vie hellénique et lul accorde une couleur et un caractère particuliers.

Comme les athlètes luttent sur le terrain pour obtenir la récompense honorifiçue, de la mème manière les musiciens, poètes, philosophes et orateurs, peintres et sculptours rivalisent entre eux avec leurs oeuvres pour "Pexcellence". Une telle attitude a ses sources essentiellement dans une conception démocratique fondamentale, affirmant que tout homme a la possibilité de se tenir face à son frère, et à revendiquer, grace à sa vertu et à sa valour, Phonneur et la premiere place. Au-delà et au-dessus de la supérionité de l'origine et de la richesse, le Grec qui croit esentiellement à légalité des droits, place la supériorité de la valeur du corps et de l'esprit, telle qu'elle se manifeste et se consacre démocratiquement dans le concours, où chacun peut participer à conditions égales, et démontrer sa vertu. Cela est témoigné par la participation aux jeux panhelléniques d'athlètes de toutes les régions de Gréce sans discrimination sociale ou généalogique, mais en vertu de la capacité de citoyen Grec libre; un même témoignage est donné par le rassemblement de savants, poètes et artistes avec les athlètes des régions les plus élolgnées, dans les grands centres de compétition de Phellénisme, à Olymple, Delphes, Isthmie, Némée. Tous se rendaient en ces endroits sacrés pour contronter leur art à celui de leurs collegues s'adonnant au meme art, devant un public exceptionnellement sensible et exigeant avec le vif espoir d'y exceller.

Dans ce règne absolu de l'esprit d'émulation, qui avait impregné toutes les actvités de l'homme, l'exploit corporel n'est pas inférieur à l'exploit intellectuel. Les deux sont à égalité. Parfois même, l'exploit corporel semble supérieur, comme l'exploit des Athèniens au combat de Marathon. Le grand poète tragique Eschyle, qui y a participé, a demandé lors de sa mort blen plus tard que solent gravés sur sa pierre funéraire non pas ses victoires pour les tragédies qu'il avait écrites, mais uniquement qu'il avait combattu a Marathon (Marathonomáchos).

Cependant, le malntien de Péquilibre dans ces deux catégories de performance corporelle et intellectuelle n'a pas duré longtemps - lidéal de la juste mesure (du motron) est une conception d'un peuple comme des Grecs, quil furent frequemment éprouvés et ébranlés justement à cause de la perte de ce métron.

Déjà, à la fin du Vième slècle av. J.C., Xénophane de Colophon stigmatise les magistrats des cttés car ils honorent plus les citoyens se distinguant pour leur musculature que les vertueux et sages. 
Et bien plus tard, avec la prédominance du professionnalisme dans Pexercice physique, Euripide écrit avec amertume: "Parmis les innombrables maux qui existent en Grèce, nul n'est pire que la race des athlètes"

Néanmoins, lidéal de la noble émulation a continué à inspirer les générations sujvantes, jusqu'aux demières années du monde ancien, et innombrables sont les enthousiastes qui ont dès lors lutté jusqu'à nos jours en vue de cultiver cet unique et savant moyen d'éducation. 


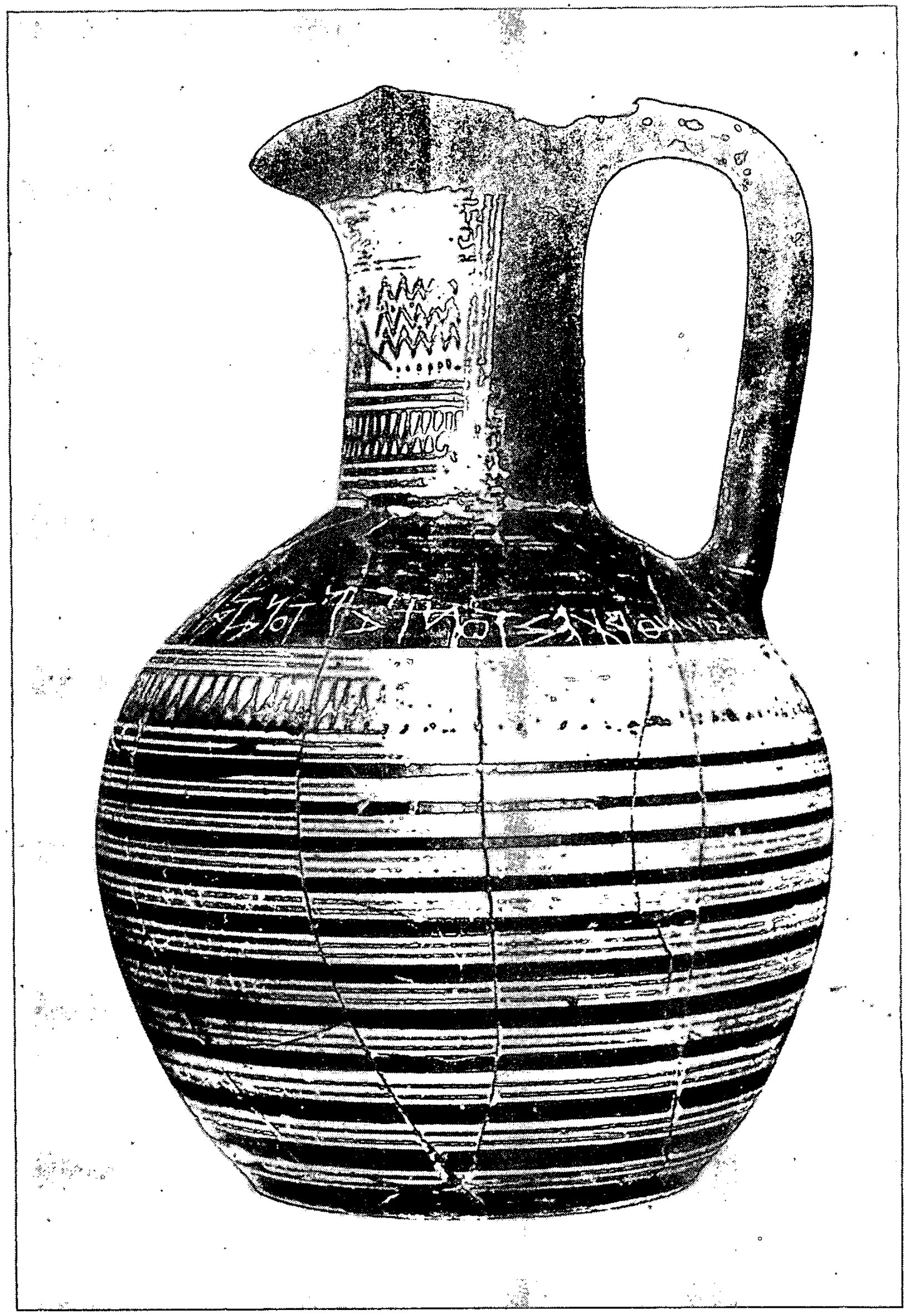

Fig. 1) Oenochoé attique de style géométrique avec inscription, provenant d'une tombe du Dipylon. (750 av. J.C.) Musée National d'Athènes. 


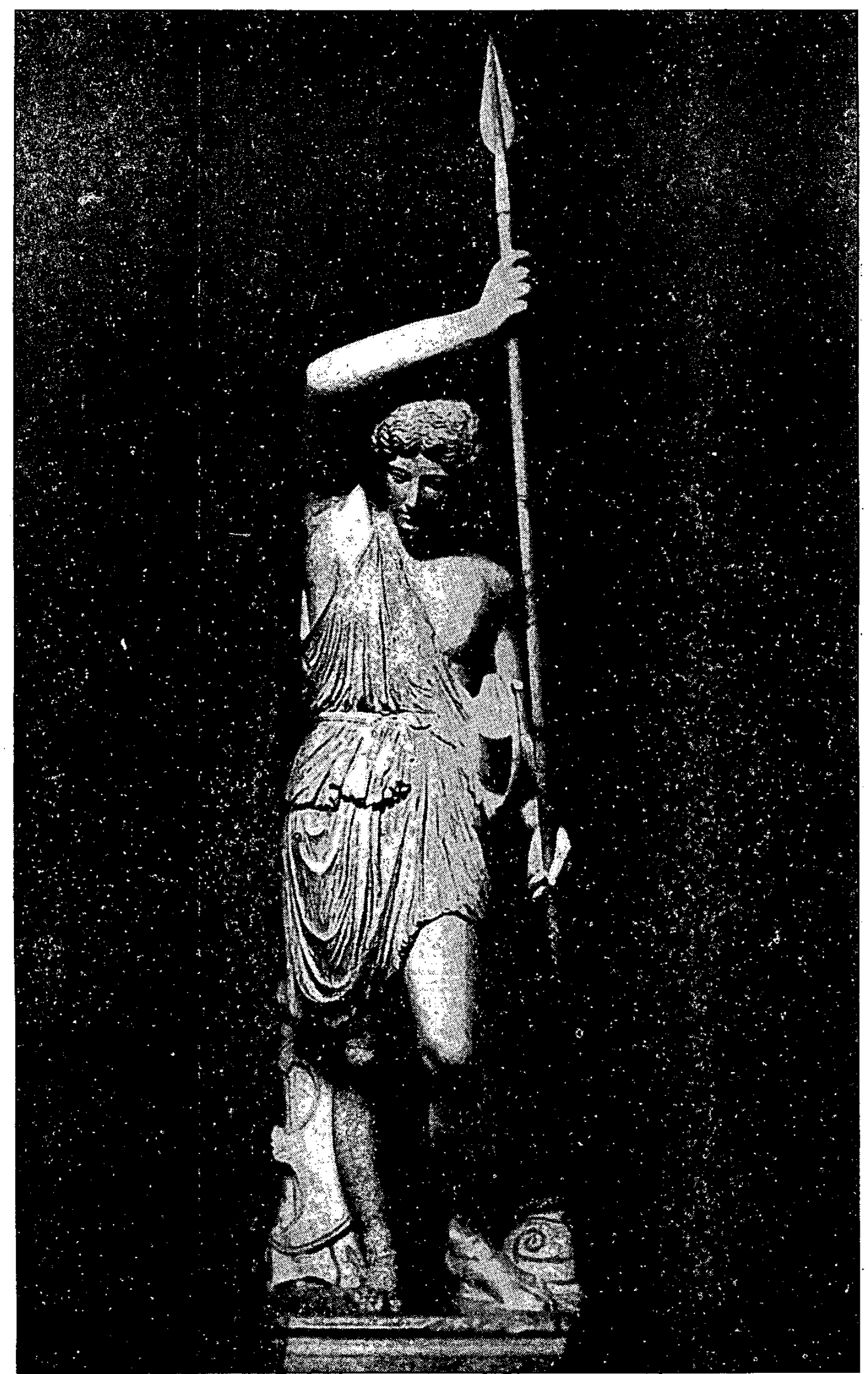

Fig. 2) Statue d'une Amazone, copie romaine d'un original de Phidias. Rome, Musée du Vatican. 


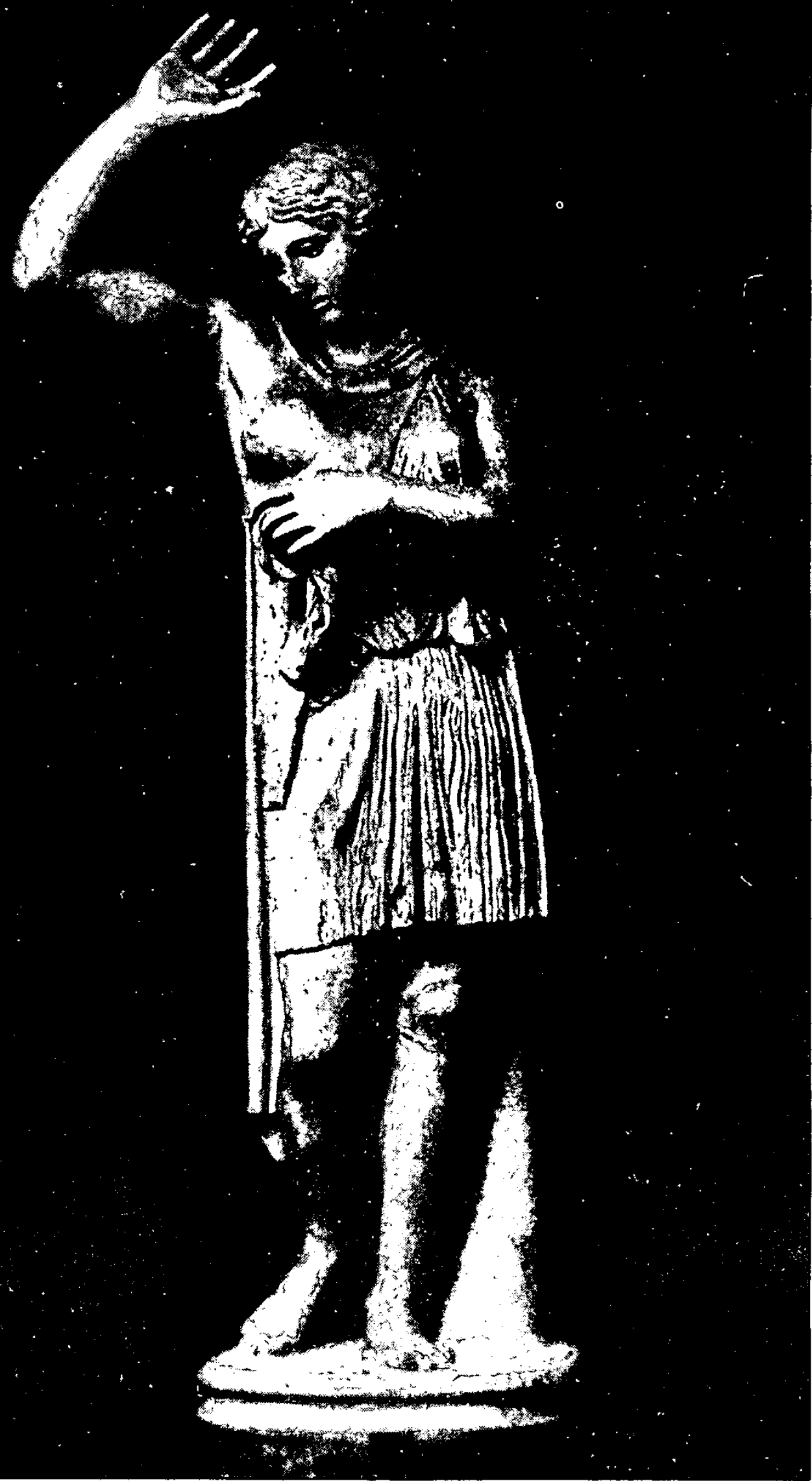

Fig.3) Statue J'une Amazone, copie romaine d'un original de Polyclète. Rome, Musée du Capitole. 


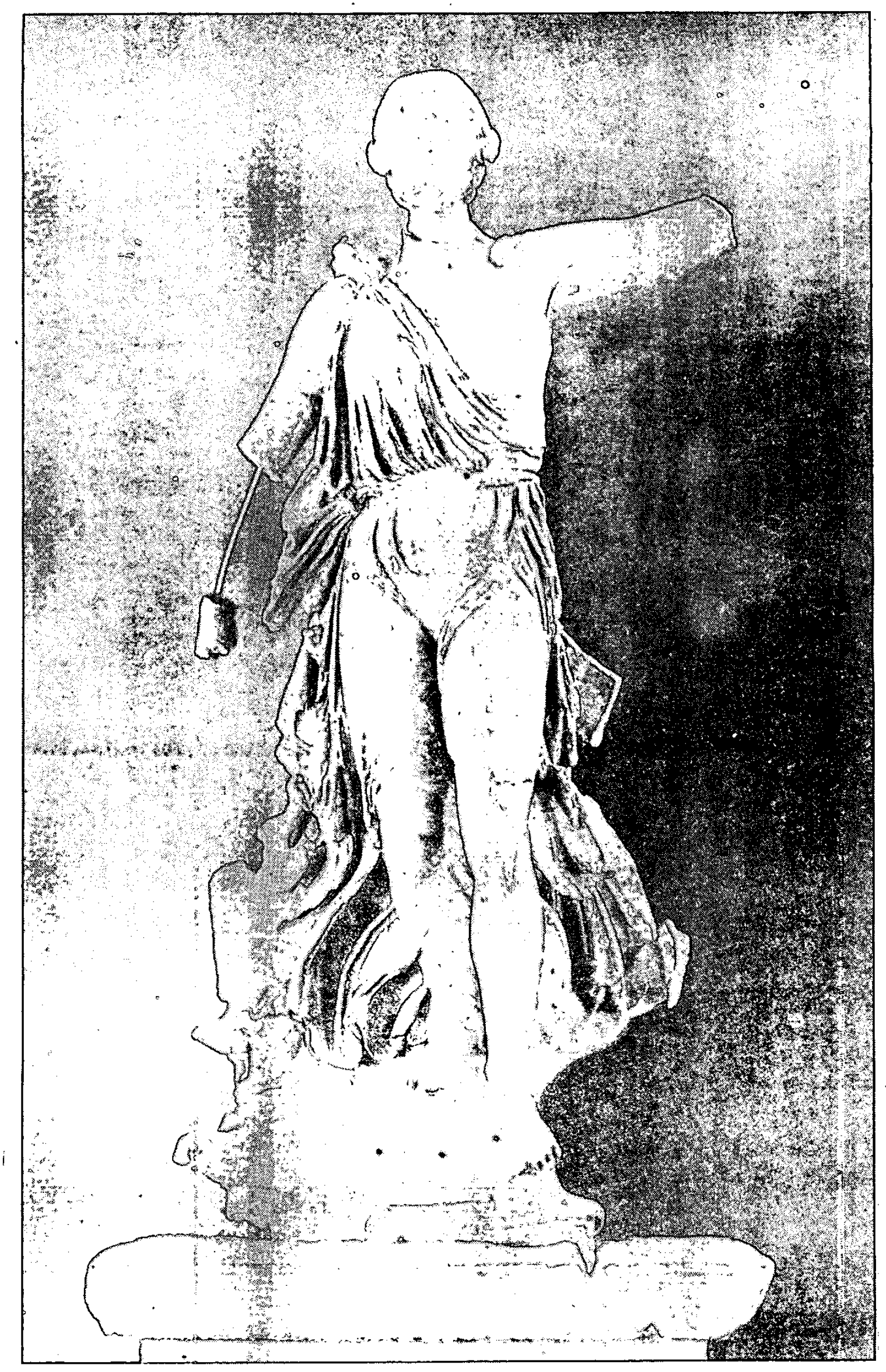

Fig.4) Niké de Péonios, dédicace des Messénies et Naupactiens après leur victoire contre les Spartiates (421 av.J.C.). Musée d'Olympie. 